\title{
- Case Report
}

\section{Crown dilaceration}

\author{
N Ghimire ${ }^{1}, \mathrm{~A} \mathrm{Rao}^{2}$ \\ ${ }^{1}$ Department of Pedodontics and Preventive Dentistry, BPKIHS, Dharan \\ ${ }^{2}$ Professor and Head, Department of Pedodontics and Preventive Dentistry \\ Manipal College of Dental Sciences, Mangalore, India
}

\begin{abstract}
Dilaceration of teeth can occur as a result of trauma to its primary predecessors. Root dilaceration is more common than crown dilacerations. A rare case of crown dilaceration of maxillary permanent central incisor has been reported in this article.
\end{abstract}

Keywords: dilaceration, permanent maxillary central incisor

\section{Introduction}

The term "dilacerations" was first coined in 1848 by Tomes, who defined the phenomenon as the forcible separation of the cap of the developed dentine from the pulp in which the development of the dentine is still progressing. ${ }^{1}$ Later, it was defined as an angulation or deviation or sharp bend or curve in the linear relationship of the crown of a tooth to its root. ${ }^{2}$ According to the glossary of dental terms, dilaceration is defined as the deformity of a tooth due to a disturbance between the unmineralized and mineralized portions of the developing tooth germ. ${ }^{3}$ Andreasen et al, in 1971, defined dilaceration as the abrupt deviation of the long axis of the crown or root portion of the tooth, which is due to a traumatic nonaxial displacement of already formed hard tissue in relation to the developing soft tissue. ${ }^{3}$

Crown dilaceration is the displacement of a portion of the developing crown at an angle to the longitudinal axis of the tooth. ${ }^{4}$ It constitutes $3 \%$ of total injuries to developing teeth and is usually due to intrusion or avulsion of their primary predecessors., ${ }^{3,4}$ Crown dilaceration usually involves the maxillary incisors and, less frequently, the mandibular incisors, because they are in close contact with the primary incisors and are more prone to injury. ${ }^{5}$ The purpose of this

Address for correspondence

Dr Neeta Ghimire, Assistant professor

Department of Pedodontics and Preventive Dentistry

BPKIHS, Dharan

Email: neetag@hotmail.com paper is to present a brief review of the literature and description of a rare case of crown dilaceration of a permanent maxillary central incisor.

\section{Case report}

A twelve year old boy reported to the Department of Pedodontics and Preventive Dentistry, with a chief complaint of pain and swelling in upper front tooth region, since 3 days. History revealed that he had episodes of pain and swelling for which medication was taken. Medical history was not contributory. Patient's father gave a history of trauma to the upper front tooth region due to fall at the age of 3 years for which no treatment was inquired.

A clinical examination revealed severe crown dilaceration of maxillary right central incisor with palatal inclination (Fig.1). The incisal and middle third of the crown had yellowish brown discoloration suggestive of enamel hypoplasia. Gingival swelling was present in relation to same tooth with pus discharging sinus.

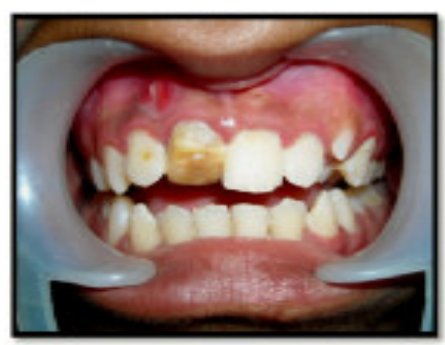

Fig.1 Dilaceration of maxillary right central incisor with palatal inclination 
Radiographic examination revealed a bend in the crown of maxillary right central incisor, giving the appearance of foreshortening coronally. Periapical radiolucency was evident with root resorption, mesially and distally (Fig 2). Other teeth were normal within the limit.

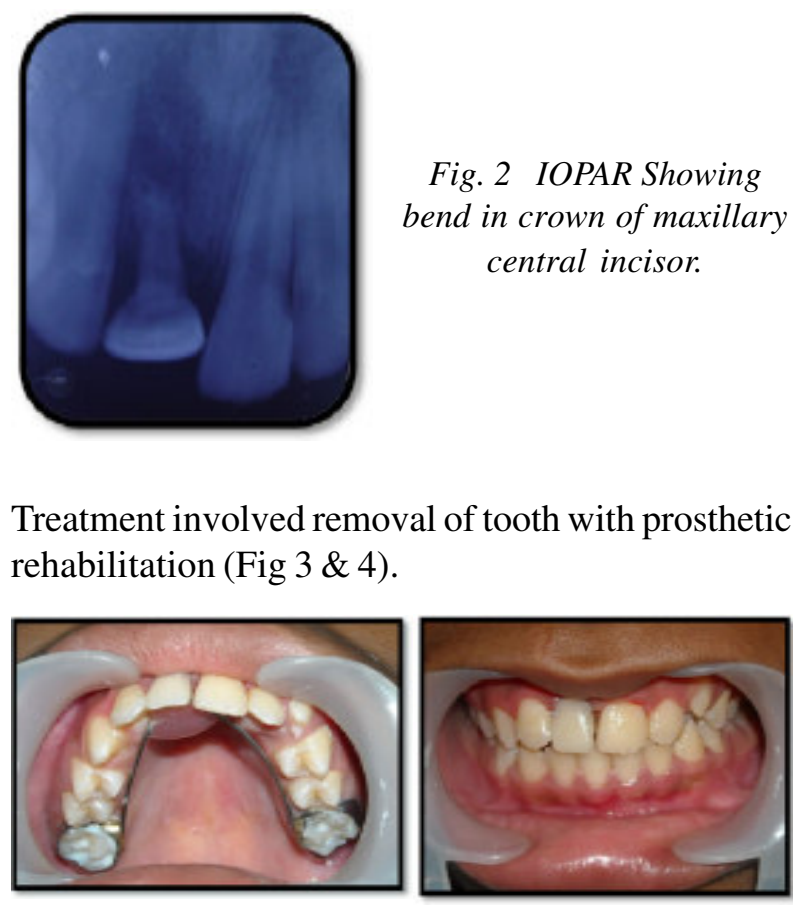

Fig. 3 \& 4: After prosthetic rehabilitation

\section{Discussion}

Crown dilaceration occur due to the traumatic non axial displacement of the already formed hard portion of the tooth in relation to the developing soft tissue portion. ${ }^{4}$ Crown dilacerations are less common than root dilacerations. ${ }^{6}$ Permanent maxillary central incisors are the most commonly dilacerated teeth. They are followed by the mandibular central and lateral incisors. ${ }^{7}$ Crown dilacerations with palatal angulation of the crown occur most commonly in upper incisors whereas labial angulation is more common in lower incisors. ${ }^{6}$ Dilaceration of primary teeth can occur due to trauma secondary to neonatal laryngoscopy and endotracheal intubation. ${ }^{7,8}$

Trauma to primary teeth can result in a wide range of disturbances to the succedaneous permanent teeth. This can range from a simple white or yellow brown discoloration to crown dilacerations, crown duplication, root dilacerations, root duplication, odontoma like malformation, partial or complete arrest of root formation, sequestration of the permanent tooth germ or disturbance in the eruption of the permanent teeth. The type and severity of the disturbance is dependent upon stage of development of permanent teeth (age at the time of injury), relationship of permanent tooth to the roots of primary teeth and direction and degree of force. ${ }^{7}$ Crown dilacerations are usually due to intrusion or avulsion of their primary predecessors. The hard tissue between a primary maxillary central incisor and its permanent successor is $<3 \mathrm{~mm}$ in thickness and mainly consists of fibrous connective tissue. ${ }^{3}$ The close anatomical relationship between the developing permanent tooth germ and root of the primary central incisor explains the severe development disorders observed in its permanent successors following strong mechanical injury to its primary predecessor. ${ }^{3,9}$ The vertical direction of the impact force is transferred in the direction of the longitudinal axis of the primary incisor and it may be transferred further up through the apex to the non calcified or partially calcified tooth germ of the permanent successor. ${ }^{3}$ Apart from trauma as a result of an accident, trauma during extraction of deciduous tooth roots can lead to dilacerations of the developing permanent premolar crowns because of their close relationship with deciduous molar tooth roots. ${ }^{6}$ Andearsen reported a dilacerations incidence of $25 \%$ in permanent teeth with developmental disturbances secondary to primary tooth injury. ${ }^{4}$ Stewart investigates 41 cases of dilacerated incisors and found that trauma accounted for only 9 cases (22\%). ${ }^{7}$ normally at the age of 2-3years, the tooth germ of the permanent maxillary incisor lies in a palatal position, above the apex of the primary incisor. If the child suffers an injury at this age, the potential consequence on the successor tooth would affect the buccal surface of its crown. ${ }^{3,9}$ In the above reported case, the trauma record showed that patient had a fall at the age of 3 years and had injury to his primary anteriors, this could be the probable reason for the dilaceration of the crown of the permanent successor.

The pathology of crown dilacerated teeth can be explained by the theory of displacement of the enamel epithelium and mineralised portion of the tooth in relation to dental papilla and cervical loop. ${ }^{7,10}$ Non axial displacement of the already formed part of the tooth can cause the following changes in relation to the dental papilla, inner and outer enamel epithelium and cervical loops. Facially, the stretched inner 
enamel epithelium is able to induce differentiation of new odontoblast and hence dentin formation is not affected. But enamel formation is usually affected. Pulp necrosis and periapical inflammation of these teeth without any decay may be a common finding. This is because the bent portion with a defective enamel and dentin (open dentinal tubules) acts as nidus for bacterial entry into pulp space. ${ }^{7}$ Brownish discoloration is caused by disturbances in the ameloblastic layer leading to defective matrix formation caused by traumatic injuries. The stretched inner enamel epithelium continues to induce differentiation of new odontoblast hence, dentin formation is not affected. ${ }^{4,7,11}$. In this case also, dilacerated maxillary incisor had a localised area of enamel discoloration and hypoplasia. Periapical pathology with root resorption was evident in radiograph.

Treatment options for crown dilacerations include: ${ }^{7}$, 12

a. Surgical exposure with or without orthodontic realignment

b. Removal of dilacerated part of crown

c. Temporary crown until root formation is complete

d. Semi or permanent restoration

e. Prosthesis or orthodontic space closure following extraction.

In this case, the dilacerated tooth was carious, root resorption was evident radiographically so, extraction was done and prosthesis was inserted. The ûxed appliance, using acrylic tooth presented in this case offers a simple and effective treatment option for the replacement of extracted tooth. It can be considered a hygienic, non-invasive, and long-term provisional treatment without bearing any risk of restricting growth, while providing superior esthetics and function. At present, there is no standard treatment procedure for the replacement of permanent anterior teeth that are lost because of trauma or caries, especially in cases that occur before cessation of growth. Following loss of the anterior tooth, it is important that an immediate replacement is provided in order to avoid esthetic, masticatory and phonetic difficulties and to maintain the edentulous space.

\section{Conclusion}

Crown dilacerations of a permanent tooth is a rare developmental anomaly usually caused by trauma to the deciduous dentition. We presented a case of crown dilaceration of maxillary central incisor.

\section{References}

1. Tomes J. A course of lectures on dental physiology and surgery delivered at the Middlesex Hospital School London: John W. Parker; 1848.

2. Shafer WG, Hine MK, Levy BM. A textbook of oral pathology. Philadelphia: WB Saunders Co; 1983.p.308-11.

3. Dilacerations of maxillary central incisor: a literature review. Dental Traumatology 2010; 26:427-433.

4. Ankur Dua, Anand C. Patil. Crown dilacerations of a permanent mandibular central incisor- a case report. Hongkong Dent J 2010; 7:36-40.

5. Prabhakar A. R, VV Reddy, Bassappa N. Duplication and dilaceration of a crown with hypercementosis of the root following trauma. Quintessence International 1998; 29: 10: 655-657.

6. Hamid Jafarzadeb, Paul V. Abbott. Dilaceration: Review of an Endodontic Challenge. J Endod 2007; 33:1025-1030.

7. Asokan S, Rayen R, Muthu M.S, Sivakumar N. Crown dilacerations of maxillary right permanent central incisor- A case report. J Indian Soc Pedod Prev Dent. 2004; 22 :( 4):197-200.

8. Seow W.K, Perham S, Young W.G et al. Dilaceration of a primary maxillary incisor associated with neonatal laryngoscopy. Journal of Pediatric Dentistry 1990; 12:321-324.

9. Diab M, El Badrawy HE. Intrusion injuries of primary incisors. Part III: Effects on the permanent successors. Quintessence Int 2000; 31:377-84.

10. Andreason FM, Andreasen JO. Textbook and color atlas of traumatic injuries to the teeth. 3rd edition. St. Louis: Mosby Co; 1994:457-94.

11. Bhushan BA, Garg S, Sharma D, Jain M. Esthetic and endosurgical management of Turner's hypoplasia; a sequelae of trauma to developing tooth germ. J Indian Soc Pedod Prev Dent 2008; 26 Suppl 3:S121-4.

12. Welbury R.R, Pediartic Dentistry. II edition Oxford Co. Page 250-253 